\title{
EVALUATION FOLLOWING TEMPOROMANDIBULAR JOINT ARTHROSCOPY WITH LYSIS AND LAVAGE- A PILOT NON-RANDOMISED STUDY
}

\author{
Arvind ${ }^{1}$, Sharad Chand ${ }^{2}$, Shadab Mohd. ${ }^{3}$, R. K. Singh 4 , U. S. Pall5, Divya Mehrortra6 ${ }^{6}$, Hari Ram ${ }^{7}$
}

${ }^{1}$ Assistant Professor, Department of Dentistry, Government Medical College, Banda, Uttar Pradesh.

${ }^{2}$ Lecturer, Department of Dentistry, Government Medical College, Banda, Uttar Pradesh.

${ }^{3}$ Professor, Department of Dentistry, KGMU, Lucknow, Uttar Pradesh.

4 Professor, Department of Dentistry, KGMU, Lucknow, Uttar Pradesh.

5 Professor, Department of Dentistry, KGMU, Lucknow, Uttar Pradesh.

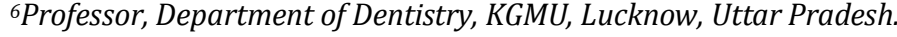

7 Professor, Department of Dentistry, KGMU, Lucknow, Uttar Pradesh.

\section{ABSTRACT}

\section{BACKGROUND}

Temporomandibular joint disorders are characterised by disturbance of mouth opening, TMJ pain and noise (clicking). The involvement of dislocation and deformation of the disc and the formation of adhesive lesions in the temporomandibular joint cavity are characteristic of this disease. With the development of the small endoscope, arthroscopy is now used for both examination and treatment of temporomandibular joint disorders.

The objective of this study was primarily aimed at investigating the efficacy of arthroscopic lysis and lavage and examination of the temporomandibular joint in patients having pain and clicking sound in temporomandibular joint.

\section{MATERIALS AND METHODS}

In this pilot, non-randomised study patients with symptoms of TMJ disease were included. Preoperative evaluation including complete history with physical examination of patients along with relevant radiograph and/or CT scan/MRI was done. Routine investigations were done. The treatment plan was carried out through Arthroscopic examination and lysis and lavage of temporomandibular joint. Patients were operated either under general anaesthesia (nasotracheal intubations) or local anaesthesia. Proper medication, physical therapy and diet were advised to patients. Outcomes were measured using pain reduction in preauricular region, reduction in clicking/grating joint sound, maximal interincisal opening, deviation of mandible and disability improvement. Patients were followed at post-operative day-1, week-1, week-2, week-4, week-8 and week-12.

\section{RESULTS}

Out of a total of six patients, arthroscopic lysis and lavage reduced pain and increasing mandibular range of motion in approximately $83 \%$ of patients. Patients aged between 20 - 30 years were the predominant age group presenting with complaints of pain clicking sound in temporomandibular joint. Females (66.6\%) were most commonly affected with pain clicking sound in temporomandibular joint.

\section{CONCLUSION}

Arthroscopic lysis and lavage is efficacious treatment modality for patients suffering and from pain clicking sound in temporomandibular joint. However, studies with larger sample size are necessary to corroborate the findings of the present study for their wider use in clinical practice.

\section{KEYWORDS}

Temporomandibular Joint, Arthroscopic Lysis.

HOW TO CITE THIS ARTICLE: Arvind, Chand S, Mohd. S, et al. Evaluation following temporomandibular joint arthroscopy with lysis and lavage - a pilot non-randomised study. J. Evolution Med. Dent. Sci. 2017;6(9):679-683, DOI: 10.14260/Jemds/2017/146

\section{BACKGROUND}

Arthroscopy of the Temporomandibular Joint (TMJ) is relatively new, the technique having been applied to this particular joint for the first time by Ohnishi in the mid 1970s. The usefulness of this method in contrast to open surgery technique is that arthroscopy offers the surgeon several

Financial or Other, Competing Interest: None.

Submission 09-01-2017, Peer Review 20-01-2017,

Acceptance 23-01-2017, Published 30-01-2017.

Corresponding Author:

Dr. Arvind,

Assistant Professor

Department of Dentistry,

Government Medical College,

Banda, Uttar Pradesh.

E-mail: njmsonline@gmail.com

DOI: $10.14260 /$ jemds $/ 2017 / 146$ effectively.[1] The level of sophistication that has been attained by the orthopaedic community in the fields of arthroscopy and arthroscopic surgery of the human knee, shoulder, elbow, ankle, hip and wrist, and continual refinement and development of new technology relating to various arthroscopic instrumentation and equipment systems has now opened up a new realm of possibilities for applying a scaled-down version of this technology in the successful diagnosis and eventual treatment of temporomandibular joint disorders.[2] Temporomandibular joint disorders are characterised by disturbance of mouth opening, TMJ pain and noise (clicking).(2) The involvement of dislocation and deformation of the disc and the formation of adhesive lesions in the temporomandibular joint cavity are 
characteristic of this disease.[2] With the development of the small endoscope, arthroscopy is now used for both examination and treatment of temporomandibular joint disorders. ${ }^{[3]}$ A study has shown that arthroscopic procedure is feasible, safe and minimally invasive, and efficacious for the treatment of patients with displacement of the disc anteriorly without reduction of the TMJ.[4] Another study has shown that Arthroscopic lysis and lavage of TMJ is safe and effective in chronic disc displacement without reduction. [5] Up to the best of our knowledge, there is no reported study from India. The goal of the present study was to evaluate efficacy of arthroscopy with intervention limited to arthroscopic examination, lavage and lysis with blunt release of adhesions when present.

\section{MATERIALS AND METHODS}

The present study was carried out in the Department of Oral and Maxillofacial Surgery of C. S. M. Medical University, Faculty of Dental Sciences, Lucknow and approved from Local Ethics Committee. Consent was obtained from all patients. A total number of 6 patients with clicking sound and pain in T.M.J. were selected. Patients underwent arthroscopic lavage and lysis using $2.4 \mathrm{~mm}$ arthroscope (Stryker Pvt. Ltd.) under Local/General Anaesthesia.

Preoperative evaluation including complete history with physical examination of patients along with relevant radiograph/CT scan/MRI was done. Routine investigations were done. The treatment plan was carried out through arthroscopic examination and lysis lavage of temporomandibular joint. Patients' complaints were reevaluated and the initial clinical examination that had been performed prior to surgery was repeated on followup visits. Patients with bony ankylosis, advanced resorption of the glenoid fossa, infection around joint area and malignant tumours were excluded. Patient at increased risk of haemorrhage and/or infection and fibrous ankylosis were also excluded. Standard TMJ arthroscope (Stryker Pvt. Ltd.) was used for this study. Patients were operated either under general anaesthesia (nasotracheal intubations) or local anaesthesia. Strict asepsis was followed and after part preparation patients were draped with sterile towels.

\section{Joint Entry Technique}

Superior temporal artery located by palpation and landmarks of temporomandibular joint and entry point to the joint space were marked. The mandibular condyle translated anteriorly by placement of large mouth prop so that interincisal distance was $30 \mathrm{~mm}$. Inj. 2\% xylocaine with adrenalin was infiltrated into area. A $21 \mathrm{G}$ needle is inserted at point A -first $5.0 \mathrm{~mm}$ at 90 to skin then $15-20 \mathrm{~mm}$ anteriorly and superiorly 45 to skin. Isotonic saline solution was injected, then there is superior temporomandibular joint space. At point $B, 2.0 \mathrm{~mm}$ vertical incision was made and superior joint space was entered as at the point $A$.

\section{Arthroscopic Examination and Lavage}

The posterior disk attachment was alternate opening and closing movement showed elongation of the PDA. Medial capsule was visualised by directing the arthroscope anteromedially. Anterior superior recess was visualised by anterolateral approach using triangulation technique. Sometimes, the lower compartment is visualised through a large disk perforation from the upper compartment.

\section{Arthroscopic Lysis and Lavage}

Arthroscopic lavage was done by $100 \mathrm{~mL}$ normal saline solution after removing arthroscope and cannula from the sheath and connecting side ports of sheath to inflow and outflow tubes for the saline solution simultaneously lysis of fibrous adhesion was done by sweeping movement of blunt probe where detected. After completion of operation betadine dressing applied on operation site. Proper postoperative care medications including antibiotics, NSAIDS, etc. prescribed. Physical therapy were started as soon as possible. Active range of motion exercises including vertical jaw opening, lateral excursions and protrusion were performed 4 - 6 times per day. Patient was placed on nonchewing diet for 2 - 4 weeks. Pressure pressing was removed after 24 hours and Neosporin ointment was applied to the puncture wounds. Patients were advised to avoid getting the wounds wet for the first 5 post-operative day.

Patient's evaluation criteria were pain reduction in preauricular region, reduction in clicking/grating joint sound, maximal interincisal opening, deviation of mandible, disability improvement. Patients were followed at day- 1 , week-1, week-2, week-4, week-8 and week-12 after the procedure.

\section{RESULTS}

Majority of patients were in the age group of 20 - 30 years. The mean age was 28.6 years. The male: female ratio was 1:2. During arthroscopic examination, fibrous band was seen in 3 joints out of total 6 joints examined. Assessment of symptoms reported by the patients as well as of clinical signs noted on the clinical examination confirms significant improvement of pain during movement and gain in mouth opening. These findings suggest that arthroscopic lysis and lavage of the temporomandibular joint is a successful modality of treatment for patients with TM Joint affliction. All six patients had history of pain in TMJ pre-operatively, which was relieved after arthroscopic lavage and lysis in all the patients and recurrence was reported in one patient after 24 weeks post-operatively. Maximal interincisal opening: in all the 6 patients, average mouth opening was $3.52 \mathrm{~cm}$ pre-operatively which became (average) $4.85 \mathrm{~cm}$ after arthroscopic lysis and lavage procedure (Table 1). Clicking sound: clicking sound was present in all 6 patients pre-operatively, which was relieved after arthroscopy (Table 2). Lysis and lavage but recurrence was reported in one patient after 24 weeks followup (Table 2). 


\begin{tabular}{|c|c|c|c|c|}
\hline Outcome & Pre-operative & Post-operative & Percent Improvement & T = 7.96 \\
\hline $\begin{array}{c}\text { Subjective assessment of pain on } \\
\text { Visual Analog Scale }\end{array}$ & $7.17 \pm 1.17(0-8)$ & $1.33 \pm 2.06(0-4)$ & $81.45 \%$ & $\mathrm{P}<.001$ \\
\hline Range of motion Mean \pm SD & $3.52 \pm 0.39$ & $4.85 \pm 0.40$ & $37.78 \%$ & $\mathrm{P}=0.003$ \\
\hline Interincisal mouth opening & & & & $\mathrm{P}<0.01$ \\
\hline Clicking sound in T.M Joint & $1.6 \pm 0.51$ & $0.51 \pm 0.40$ & $54.64 \%$ & \\
\hline Disability improvement & $1.83 \pm 0.75$ & $0.83 \pm 1.16$ & 60 & 0 \\
\hline $\begin{array}{c}\text { Deviation of mandible during mouth } \\
\text { opening present Number of patients }\end{array}$ & 05 & 02 & 1 & \\
\hline Locking of jaw/Subluxation & 1 & \multicolumn{2}{|c|}{} \\
\hline \multicolumn{7}{|c|}{ Table 1. Outcome Analysis } \\
\hline
\end{tabular}

\begin{tabular}{|c|c|c|c|c|c|c|c|}
\hline Name & $\begin{array}{c}\mathbf{1} \\
\text { week }\end{array}$ & $\begin{array}{c}\mathbf{4} \\
\text { Weeks }\end{array}$ & $\mathbf{1 2}$ Weeks & 24 Weeks & $\begin{array}{c}\text { 1 Week Vs 4 } \\
\text { Weeks } \\
\text { P Value }\end{array}$ & $\begin{array}{c}\text { 1 Week Vs } \\
\text { 12 Weeks Week Vs } \\
\text { P Value }\end{array}$ & $\begin{array}{c}\text { 24 Weeks } \\
\text { P Value }\end{array}$ \\
\hline $\begin{array}{c}\text { Post-operative monitoring of } \\
\text { pain Mean } \pm \text { S.D }\end{array}$ & $1.66 \pm 1.03$ & $0.33 \pm 0.81$ & $0.5 \pm 1.22$ & $0.66 \pm 1.63$ & 0.010 & 0.033 & 0.11 \\
\hline M .I. Opening (in cms) & $3.9 \pm 0.43$ & $4.6 \pm 0.19$ & $5.01 \pm 0.39$ & $4.8 \pm 0.46$ & 0.21 & 0.0009 & 0.004 \\
\hline Clicking sound & 1 & 0 & 1 & 1 & & & \\
\hline Swelling & 4 & 0 & 0 & 0 & & & \\
\hline Deviation of mandible & 1 & 1 & 1 & 2 & & & \\
\hline Disability & 0 & 2 & 3 & 4 & & \\
\hline
\end{tabular}

\section{DISCUSSION}

The arthroscopy is most recently introduced surgical treatment of the TMJ first described by Ohnishi ${ }^{6}$ in 1975 . The usefulness of this method in contrast to open surgery technique is that arthroscopy offers the surgeon several advantages in investigating and thereby improving the diagnosis and treatment in patients with TMJ disorders more effectively.

After surgical arthroscopy peaked in popularity in the early 1990s, it has since waned in recent years as few surgeons are prepared to invest the extra time and effort towards upgrading their skills to undertake surgical arthroscopy procedures. This is largely because surgical arthroscopy has failed to provide any firm evidence that the results are superior to simple arthroscopic lavage and lysis. In other words, it is difficult to justify patients being subjected to the added expense and increased potential morbidity of surgical arthroscopy when the literature does not support its role as being superior to that of lavage and lysis.

In this study, the mean age of the patients at surgery was 28.6 years which corresponds to the study by Sorel et al ${ }^{7}$ who reported the mean age being 28 years and 4 months.

There seemed to be an over representation of TMJ disorders in women in the second and third decade in this study. The reasons for the higher prevalence of TMJ disc displacement in women and the over representation of females with orofacial pain remain obscure. A hypothesis has been put forward that women's threshold for pain are lower than men and that women therefore more readily seek treatment for illness than men. According to the American Society of Temporomandibular Joint Surgeons, 8 the prevalence of Internal Derangement in males and females is not clearly defined, but occurs most often in females. Women also comprise about $80 \%$ of patients seeking treatment for joint pain. The average age of patients seeking surgical care is nearly 30 years. This is similar to our findings, as most patients in our study were in age group of $20-30$ years and mean age was 28.6 years and females (66\%) outnumbered the males.

According to American Society of Temporomandibular Joint Surgeons, ${ }^{8}$ studies evaluating the efficacy of arthroscopic lysis and lavage for the treatment of internal derangement reported success rates ranging from $80 \%$ to 86\%. Sanders (1986) ${ }^{9}$ and Davis et al (1991) ${ }^{10}$ also reported post-arthroscopy success rates between 93\% to 100\%; however, each of these studies selected patients from one diagnostic category (ADDW) for one specific treatment (arthroscopy) without the use of any control or competing experimental group. In a multicentre study involving 4831 joints, $94.3 \%$ good or excellent reduction in disability was observed which is similar (83.3\%) to our study after arthroscopic lysis and lavage. ${ }^{11}$ In another retrospective study on arthroscopic lavage and lysis by Indresano et al, ${ }^{12}$ disability was reduced by $86 \%$ after arthroscopic lysis and lavage, which is again comparable to our study. Kurita et al $19988^{13}$ reported a success rate of $86 \%$ after arthroscopic lysis and lavage, which is comparable to our study. Israel et al ${ }^{14}$ reviewed 11 studies of TMJ arthroscopic outcomes published between 1987 and 1996 in a cumulative total of 6,071 joints in 3,955 patients, a mean success rate of $84 \%$ was calculated, which is comparable to the success rate $(83.3 \%)$ reported in our study. Murakami et al 15 compared conservative treatment, arthrocentesis and arthroscopy in 3 groups of patients with closed lock and reported that arthroscopy $(91 \%$ success rate) and arthrocentesis (70\% success rate) were far superior to conservative measures alone ( $56 \%$ success rate). This is slightly greater than our study results. Smolka et al ${ }^{16}$ also reported the overall success rate of $78.3 \%$, which is again similar (83.3\%) to the rate reported in our study.

The reduction of pain levels was evaluated using a VAS for pain. The treatment was considered successful when pain levels of less than 2 to 3 were achieved post-operatively. Mean reduction of pain in our study was 5.84, which is greater than that $(3.8 \pm 0.45)$ reported by Goudot et al ${ }^{17}$ where out of 152 joints treated with the method, $92 \%$ 
experienced a significant reduction of pain and restoration of mandibular function.

In another study by Indresano,12 pain was reduced by $81 \%$ after arthroscopic and lavage. Of all 6 patients who presented with pain arising from the affected TMJ in this study, the mean preoperative pain score was $7.17 \pm 1.17$ as measured on a visual analog scale (range, 0 to 10$)$. The mean pain score 24 weeks after arthroscopy was $1.33+2.06(0-4)$, which is an improvement of 5.84. This equates to an average visual analog scale of $81.45 \%$ reduction in measurement of pain levels after TMJ arthroscopy. One of the 6 patients, however, reported recurrence of pain levels after 2 weeks of TMJ arthroscopy. Post-operative physiotherapy helped to maintain the reduced pain levels and rarely led to any further significant improvement. This corresponds to the study by Dimitroulis et al 17 who reported pain arising from the affected TMJ among $76.8 \%$ patients and the mean preoperative pain score to be $7.1 \pm 1.9$ as measured on a visual analog scale (range, 0 to 10). The mean pain score 6 weeks after arthroscopy was $2.4 \pm 1.0$, which was an improvement of $4.7 \pm 0.9$. This equated to an average visual analog scale of $66.2 \%$ reduction in measurement of pain levels after TMJ arthroscopy.

Hani Abd-Ul-Salam et al ${ }^{18}$ observed pain on the Visual Analogue Scale preoperatively ranged from 6 to 10 and postoperatively ranged from 2 to 6 with a mean gain of 5.6, which is $63.63 \%$ being lower in comparison to our study (81.45\%).

In terms of pain scores on the visual analog scale of 0 to 10 , Israel et al ${ }^{14}$ also observed a mean reduction of 4.6 units, which is again in close agreement with this study, which recorded 5.84 units. Our study found that $83 \%$ of patients reported an improvement in their pain levels after arthroscopy, which is almost similar than the average of $82 \%$ reported by Israel et al among the 11 studies reviewed by them. However, direct comparisons with this study cannot be made because of the significant variability in diagnosis and arthroscopic techniques used in the 11 studies reported by them.

The mean preoperative maximum interincisal opening was $3.52 \pm 0.39 \mathrm{~cm}$. One week after arthroscopy, the mean interincisal opening was $4.6 \pm 0.19 \mathrm{~cm}$, whereas at 24 weeks after arthroscopy the mean interincisal opening was $4.85 \pm$ $0.40 \mathrm{~cm}$. Therefore, the mean improvement in mouth opening at 24 weeks after arthroscopy was $1.33 \mathrm{cms}$. All the 6 patients (100\%) experienced improvement in maximum mouth opening after arthroscopy.

These findings correspond to those reported by Dimitroulis et $\mathrm{al}^{17}$ with mean preoperative maximum interincisal opening $25.5 \pm 5.4 \mathrm{~mm}$. They observed that the difference between pre-operative $(24.6 \mathrm{~mm})$ and postoperative $(42.3 \mathrm{~mm})$ opening was $17.7 \mathrm{~mm}$. Abd-Ul-Salam et al18 reported maximal vertical mouth-opening preoperatively ranged from $6 \mathrm{~mm}$ to $40 \mathrm{~mm}$, which increased post-operatively, ranged from $30 \mathrm{~mm}$ to $53 \mathrm{~mm}$ and mean gain of $10.9 \mathrm{~mm}$ which is comparable to our study. Goudot et al ${ }^{19}$ observed a $9.6 \mathrm{~mm}$ mean gain in mouth opening after arthroscopy, which is lower than our study.

Sorel et $\mathrm{al}^{7}$ measured the total opening movement of the mandible using interincisal distance plus the vertical dental overbite and observed a mean increase of $8.1 \mathrm{~mm}$.
Israel et $\mathrm{al}^{14}$ also reported a mean improvement in interincisal opening of $10.4 \mathrm{~mm}$, which compares closely with the $13.3 \mathrm{~mm}$ reported in the present study.

Left and/or right deviation on opening was present in 5 patients prior to surgery and 2 patients on followup, $60 \%$ improvement was noted in our study, which is lower than that (73\% improvement) reported by Sorel et al.7 Synovitis and hyperaemia were present in more than two-thirds of the joints $(84 \%)$ in our study, while Holmlund et al ${ }^{20}$ found that 93\% of the TMJs that underwent arthroscopy had evidence of synovitis. Synovitis and hyperaemia were found in more than half the joints (54\%) examined in the study of Dimitroulis et al. ${ }^{17}$ The clinical significance of synovitis and hyperaemia is because of TMJ pain.

Adhesions were found in $50 \%$ of all joints examined in our study, which is higher than those reported by Rosenberg21 (38\%) and Dimitroulis et al ${ }^{17}$ (38\%). This suggests that adhesions are not necessarily the primary or essential cause of chronic pain and clicking sound in TMJ, although it has been noted that a large number of intraarticular adhesions, which were not found in this group of patients may be considered as fibrous ankylosis.

The rarity of temporomandibular joint disorders in the older population has long been pointed out as an argument against extensive intervention in the generally young patients affected by these problems. Some recent studies have tried to investigate this further and have supported the value of time as a healing factor. This would of course influence the results of a study such as the present one, where a lot of the longterm benefit observed might have little to do with the technique itself, but rather with the passage of time.

There would, however, still be good reasons to look carefully at surgically conservative techniques such as arthroscopy and arthrocentesis. For one if a simple treatment with low morbidity can relieve the patients enough to ensure a more normal function and decrease the long-term consumption of multiple medications, while the tincture of time takes effect it deserves serious consideration. It can also be very difficult to avoid any surgical intervention in a patient with severe pain. The possibility of utilising a procedure that is satisfying for the patient, while avoiding the possibility of starting the cycle of the multiply-operated temporomandibular joint patient, should also appeal to the surgeon.

There has been no possibility, however, of studying the very long-term effect (after 20 - 30 years) of these conservative measures, but one can doubt serious sequelae from these surgeries by themselves. This study supports the beneficial effect of arthroscopy with lysis and lavage for the treatment of chronic pain in the temporomandibular joint. However, to come at a definite conclusion, the study should have a large sample size and a longer followup.

\section{CONCLUSION}

Arthroscopic lysis and lavage is an efficacious treatment modality for patients suffering and from pain clicking sound in temporomandibular joint. However, studies with larger sample size are necessary to corroborate the findings of the present study for their wider use in clinical practice. 


\section{REFERENCES}

[1] Al-Moraissi EA. Arthroscopy versus arthrocentesis in the management of internal derangement of the temporomandibular joint: a systematic review and meta-analysis. Int J Oral Maxillofac Surg 2015;44(1):104-12.

[2] Prasad SR, Kumar NR, Shruthi HR, et al. Temporomandibular pain. J Oral Maxillofac Pathol 2016;20(2):272-5.

[3] Moses JJ, Poker ID. Temporomandibular joint arthroscopy: the endaural approach. Int J Oral Maxillofac Surg 1989;18(6):347-51.

[4] Zhu Y, Zheng C, Deng Y, et al. Arthroscopic surgery for treatment of anterior displacement of the disc without reduction of the temporomandibular joint. Br J Oral Maxillofac Surg 2012;50(2):144-8.

[5] Machoň V, Sedý J, Klíma K, et al. Arthroscopic lysis and lavage in patients with temporomandibular anterior disc displacement without reduction. Int J Oral Maxillofac Surg 2012;41(1):109-13.

[6] Onishi M. Arthroscopy of the temporomandibular joint (author's transl). Journal of Japanese Stomatological Association 1975;42:207-13.

[7] Sorel B, Piecuch JF. Long-term evaluation following temporomandibular joint arthroscopy with lysis and lavage. Int J Oral Maxillofac Surg 2000;29(4): 259-63.

[8] American Society of Temporomandibular Joint Surgeons. Guidelines for diagnosis and management of disorders involving the temporomandibular joint and related musculoskeletal structures. Cranio 2003;21(1):68-76.

[9] Sanders B. Arthroscopic surgery of the temporomandibular joint: treatment of internal derangement with persistent closed lock. Oral Surg Oral Med Oral Pathol 1986;62(4):361-72.

[10] Davis CL, Kaminishi RM, Marshall MW. Arthroscopic surgery for treatment of closed lock. J Oral Maxillofac Surg 1991;49(7):704-7.

[11] McCain JP, Sanders B, Koslin MG, et al. Temporomandibular joint arthroscopy: a 6-year multicenter retrospective study of 4,831 joints. J Oral Maxillofac Surg 1992;50(9):926-30.
[12] Indresano AT. Surgical arthroscopy as the preferred treatment for internal derangements of the temporomandibular joint. J Oral Maxillofac Surg 2001;59(3):308-12.

[13] Kurita K, Goss AN, Ogi N, et al. Correlation between preoperative mouth-opening and surgical outcome after arthroscopic lysis and lavage in patients with disc displacement without reduction. J Oral Maxillofac Surg 1998:56(12):1394-7.

[14] Israel HA. Part I: the use of arthroscopic surgery for treatment of temporomandibular joint disorders. J Oral Maxillofac Surg 1999;57(5):579-82.

[15] Murakami K, Moriya Y, Goto K, et al. Four-year followup study of temporomandibular joint arthroscopic surgery for advanced stage internal derangements. J Oral Maxillofac Surg 1996;54(3):285-91.

[16] Smolka W, Lizuka T. Arthroscopic lysis and lavage in different stages of internal derangement of the temporomandibular joint: correlation of preoperative staging to arthroscopic findings and treatment outcome. J Oral Maxillofac Surg 2005;63(4):471-8.

[17] Dimitroulis G. A review of 56 cases of chronic closed lock treated with temporomandibular joint arthroscopy. J Oral Maxillofac Surg 2002;60(5):519-525.

[18] Abd-Ul-Salam H, Weinberg S, Kryshtalskyj B. The incidence of reoperation after temporomandibular joint arthroscopic surgery: a retrospective study of 450 consecutive joints. Oral Surg Oral Med Oral Pathol Oral Radiol Endod 2002;93(4):408-11.

[19] Goudot $P$, Jaquinet AR, Hugonnet $S$, et al. Improvement of pain and function after arthroscopy and arthrocentesis of the temporomandibular joint: a comparative study. J Cranio-Maxillo-fac Surg 2000;28(1):39-43.

[20] Holmlund A, Gynther G, Axelsson S. Efficacy of arthroscopic lysis and lavage in patients with chronic locking of the temporomandibular joint. Int J Oral Maxillofac Surg 1994;23(5):262-5.

[21] Rosenberg I, Goss AN. The outcome of arthroscopic treatment of temporomandibular joint arthropathy. Aust Dent J 1999;44(2):106-11. 